(C) 1998 IEEE. Personal use of this material is permitted. Permission from IEEE must be obtained for all other uses, in any current or future media, including reprinting/republishing this material for advertising or promotional purposes, creating new collective works, for resale or redistribution to servers or lists, or reuse of any copyrighted component of this work in other works. 


\title{
Performance Characteristics of a Compact Position-Sensitive LSO Detector Module
}

\author{
Juan J. Vaquero, Jürgen Seidel, Stefan Siegel, Member, IEEE, William R. Gandler, Senior Member, IEEE, \\ and Michael V. Green*
}

\begin{abstract}
We assembled a compact detector module comprised of an array of small, individual crystals of lutetium oxyorthosilicate:Ce (LSO) coupled directly to a miniature, metal-can, position-sensitive photomultiplier tube (PSPMT). We exposed this module to sources of 511-keV annihilation radiation and beams of 30- and 140-keV photons and measured spatial linearity; spatial variations in module gain, energy resolution, and event positioning; coincidence timing; the accuracy and sensitivity of identifying the crystal-of-first-interaction at $511 \mathrm{keV}$; and the effects of intercrystal scatter and LSO background radioactivity. The results suggest that this scintillator/phototube combination should be highly effective in the coincidence mode and can be used, with some limitations, to image relatively low-energy single photon emitters.

Photons that are completely absorbed on their first interaction at $511 \mathrm{keV}$ are positioned by the module at the center of a crystal. Intercrystal scatter events, even those that lead to total absorption of the incident photon, are placed by the module in a regular "connect-the-dot" pattern that joins crystal centers. As a result, the accuracy of event positioning can be made to exceed $\mathbf{9 0 \%}$, though at significantly reduced sensitivity, by retaining only events that occur within small regions-of-interest around each crystal center and rejecting events that occur outside these regions in the connect-the-dot pattern.
\end{abstract}

Index Terms-Intercrystal scatter, lutetium oxyorthosilicate:Ce (LSO), position-sensitive photomultiplier tubes, small animal positron emission tomography (PET).

\section{INTRODUCTION}

C OMPACT detector modules are useful, or required, in a variety of imaging applications ranging from imaging probes [1]-[4] to small animal PET scanners [5]-[9]. For small animal PET, such modules must also be capable of very accurately locating the endpoints of lines-of-response in space while simultaneously possessing high sensitivity to $511-\mathrm{keV}$ radiation, high count rate capability, and good random coincidence rejection. Watanabe et al. [10] have sought these goals by directly coupling arrays of individual $2.8 \times 6.8 \times 30 \mathrm{~mm}^{3}$ BGO crystals to miniature, metal-can PSPMT's. Cherry et al.

Manuscript received January 30, 1998; revised June 30, 1998. The work of J. J. Vaquero was supported in part by a grant from CICYT (Spanish Government). The work of S. Siegel was supported by a grant from the National Research Council. The Associate Editor responsible for coordinating the review of this paper and recommending its publication was C. Thompson. Asterisk indicates corresponding author.

J. J. Vaquero, J. Seidel, S. Siegel, and W. R. Gandler are with the Nuclear Medicine Department, National Institutes of Health, Bethesda, MD 20892 USA.

*M. V. Green is with the Nuclear Medicine Department, National Institutes of Health, Building 10, Room 1C401 MSC 1180, Bethesda, MD 20892 USA (e-mail: mike@nmdhst.cc.nih.gov).
[8] have achieved even higher performance by coupling arrays of individual $22^{2} 10 \mathrm{~mm}_{3}$ crystals of the newly available scintillator lutetium ðxyorthosilicate:Ce (LSO) to multichannel photomultiplier tubes through light guides. However, while both of these modules exhibit performance characteristics substantially better than those in conventional human PET scanners, the use of BGO in the firs module and light guides in the second results in a reduction of useful scintillation light compared to other possible scintillator/phototube coupling schemes.

In order to maximize both the amount of light and the efficienc of collection while retaining the advantages of a compact design, we assembled a detector module composed of LSO crystals coupled directly to a miniature metal-can PSPMT. Here, we report selected performance characteristics of this module when exposed to single photon beams and when illuminated by positron annihilation radiation.

\section{Materials AND MethodS}

\section{A. Detector Modules}

LSO was obtained in bulk from CTI (Knoxville, TN). LSO is a scintillator with high density $(7.4 \mathrm{~g} / \mathrm{cc})$, high light output [55\%-75\% of $\mathrm{NaI}(\mathrm{Tl})]$, high linear attenuation coefficien $(0.86 / \mathrm{cm}$ at $511 \mathrm{keV})$, and a short light decay time $(40 \mathrm{~ns})$ [11]. LSO is rugged and not hygroscopic but is radioactive (approximately 300 disintegrations/s/cc of LSO [12]) due to the presence of Lu-176. The bulk LSO was cut into individual crystal elements $2 \mathrm{~mm} \quad 2 \mathrm{~mm} \quad$ 10-mm long (3D Precision Optics, Ravenna, $\mathrm{OH}$ ). Eåch crysłal was mechanically polished on all surfaces except the entrance end which was finel ground. All crystals were individually double-wrapped with white, diffusely reflectin Teflo tape including the entrance end [8]. Eighty-one of these prepared crystals were then packed together in a plastic retainer to form a 99 array (Fig. 1). The pitch of this array was approximately $2.2 \mathrm{~mm}$.

This array was then coupled with optical grease (compound V-788, 1.47, Visilox Systems, Troy, New York) to a miniature, $\overline{\text { metal }}$ can, position-sensitive photomultiplier tube (Hamamatsu R5900U-00-C8). Amplificatio in these tubes is accomplished with 11 metal channel dynodes. Position readout of the tube was performed by collecting current from two crossed anode layers, each layer consisting of four side-by-side anode plates separated by $0.5 \mathrm{~mm}$ [13]. Since the distribution of current across the four anode plates in a layer depends on the location of the scintillation light flas within the field-of 


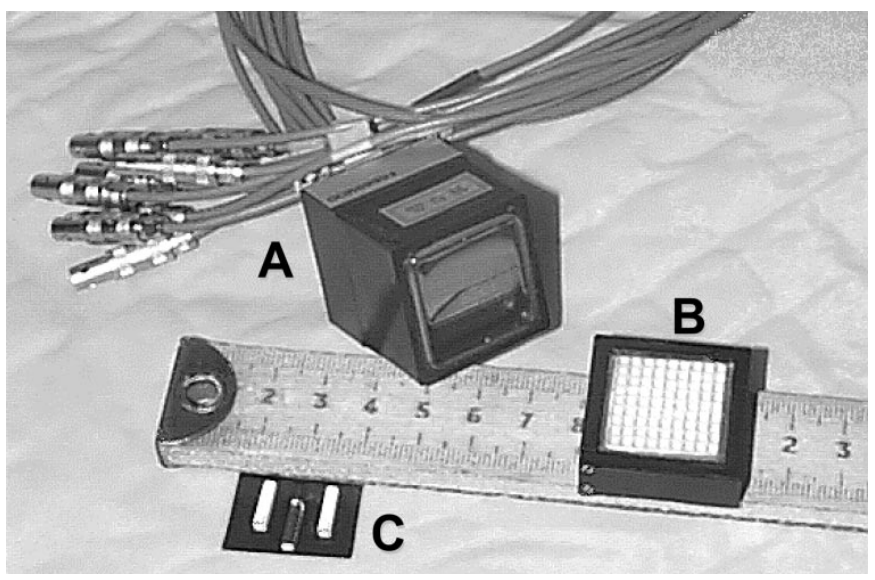

Fig. 1. Components of the LSO/PSPMT detector module (a) Hamamatsu R5900U-00-C8 metal-can PSPMT; (b) $9 \times 9$ LSO crystal array in plastic frame holder; (c) three LSO crystals, two wrapped with Teflo tape. Scale is in centimeters.

view (FOV) of the tube, the relative magnitudes of the anode currents within each layer can be used to determine the crystalof-interaction within the LSO array, i.e., current collected from the four anodes in one layer determines the $X$-coordinate of the event and current from the four anodes in the second layer identifie the $Y$-coordinate of the event.

In operation, light from a scintillation event in an LSO crystal is directed onto the thin $(1.6 \mathrm{~mm},[10])$ glass entrance window of the tube, an electron cloud is emitted from the photocathode of the tube, amplified and collected on the four $X$ anode plates and four $Y$ anode plates. Thus, a total of eight position signals (four $X$ anode signals and four $Y$ anode signals) are collected for each event. An additional signal from the last dynode is used for timing and coarse energy discrimination.

The tube has a 1.4-ns anode pulse rise time [13] well suited to the fast light rise and decay times of LSO [14]. Spectral response ranges from $300-650 \mathrm{~nm}$ with a maximum at 420 $\mathrm{nm}(20 \%$ quantum efficiency) the same wavelength as the maximum of the LSO emission spectrum [13]. The useful FOV (UFOV) of the tube is approximately $20 \mathrm{~mm} \times 20 \mathrm{~mm}$. The LSO array and the tube (Fig. 1) were housed in a thin walled, light-tight aluminum box.

\section{B. Data Acquisition}

Without preamplification all eight position signals for each scintillation event were digitized by charge integrating (200-ns integration time) analog-to-digital converters (ADC's) (LeCroy FERA 4300B) whenever the signal from the last dynode, boosted with a fast amplifie (EG\&G Ortec VT-120), exceeded a low threshold set just above the dynode electronic noise level. For measurements at different photon energies, the high-voltage applied to the PSPMT was adjusted such that the magnitudes of the eight position signals spanned approximately $80 \%$ of the dynamic range of the ADC's. Delay lines between the tube and the ADC's adjusted the relative timing of the trigger and position signals. These digitized signals were acquired into an intermediate dual buffer memory (LeCroy FERA 4302) and then transferred to the host computer (Macintosh PowerPC 9500/180MP) via a SCSI controller (Jorway 73A) and recorded in "list" mode.

Coincidence timing was established by connecting the amplifie output of the last dynode of the detector module and that of a second LSO detector, to discriminators (LeCroy 821). The discriminator outputs were, in turn, connected to a time-toamplitude converter (TAC). The TAC spectrum time axis was calibrated by placing an additional time delay of known length between one dynode output and the TAC and observing the time shift of the LSO TAC spectrum relative to the unshifted spectrum. Based on these TAC measurements, the coincidence timing window for the experiment described below was set to twice the measured full-width-at-half-maximum (FWHM) width of the frequency distribution of arrival times.

\section{Data Processing}

The $X, Y$ location of an event within the UFOV was computed as

$$
\begin{aligned}
& X=\frac{\left[a_{1} \cdot x_{1}+a_{2} \cdot x_{2}+a_{3} \cdot x_{3}+a_{4} \cdot x_{4}\right]}{\left[a_{1}+a_{2}+a_{3}+a_{4}\right]} \\
& Y=\frac{\left[b_{1} \cdot y_{1}+b_{2} \cdot y_{2}+b_{3} \cdot y_{3}+b_{4} \cdot y_{4}\right]}{\left[b_{1}+b_{2}+b_{3}+b_{4}\right]} .
\end{aligned}
$$

Here, $x_{i}$ and $y_{i}$ are the physical coordinates of the $X$ and $Y$ anode plates in the PSPMT in millimeters, and $a_{i}, b_{i}$ are the magnitudes of the (four) $X$ anode and (four) $Y$ anode plate signals for the event. This calculation was performed only for events where all position signals were within the dynamic range of the ADC's. Exclusion of events above the dynamic range of the ADC's is equivalent to placing an upper level hardware discriminator in the acquisition chain that rejects high-energy events associated with the LSO background. True energy windowing involved placement of upper and lower software thresholds within the energy range corresponding to the dynamic range of the ADC's.

Apparent gain varies with position across the face of the detector module, generally being higher at the center than at the edges of the UFOV. Thus, no global upper or lower energy threshold can be applied to events occurring at different locations within the UFOV. To make the gain appear uniform across the UFOV and allow the same upper and lower energy thresholds to be used everywhere within the UFOV, energy spectra were acquired for each crystal during fiel floo illumination of the detector module with $511-\mathrm{keV}$ radiation. The channel number of the $511-\mathrm{keV}$ photopeak maximum in each of these 81 spectra was identifie and the mean of these 81 values computed. A scale factor was then created for each crystal by calculating the ratio of this mean to the channel number of each individual photopeak maximum. These scale factors, one for each LSO crystal, were stored in a lookup table for later use. Subsequent events were corrected for gain variations by multiplying the sum of all eight anode signals for the event (the energy of the event) by the scale factor associated with the crystal in which the event occurred. This gain-adjusted sum was then compared against the (single) upper and lower energy thresholds set by the user. If the scaled 


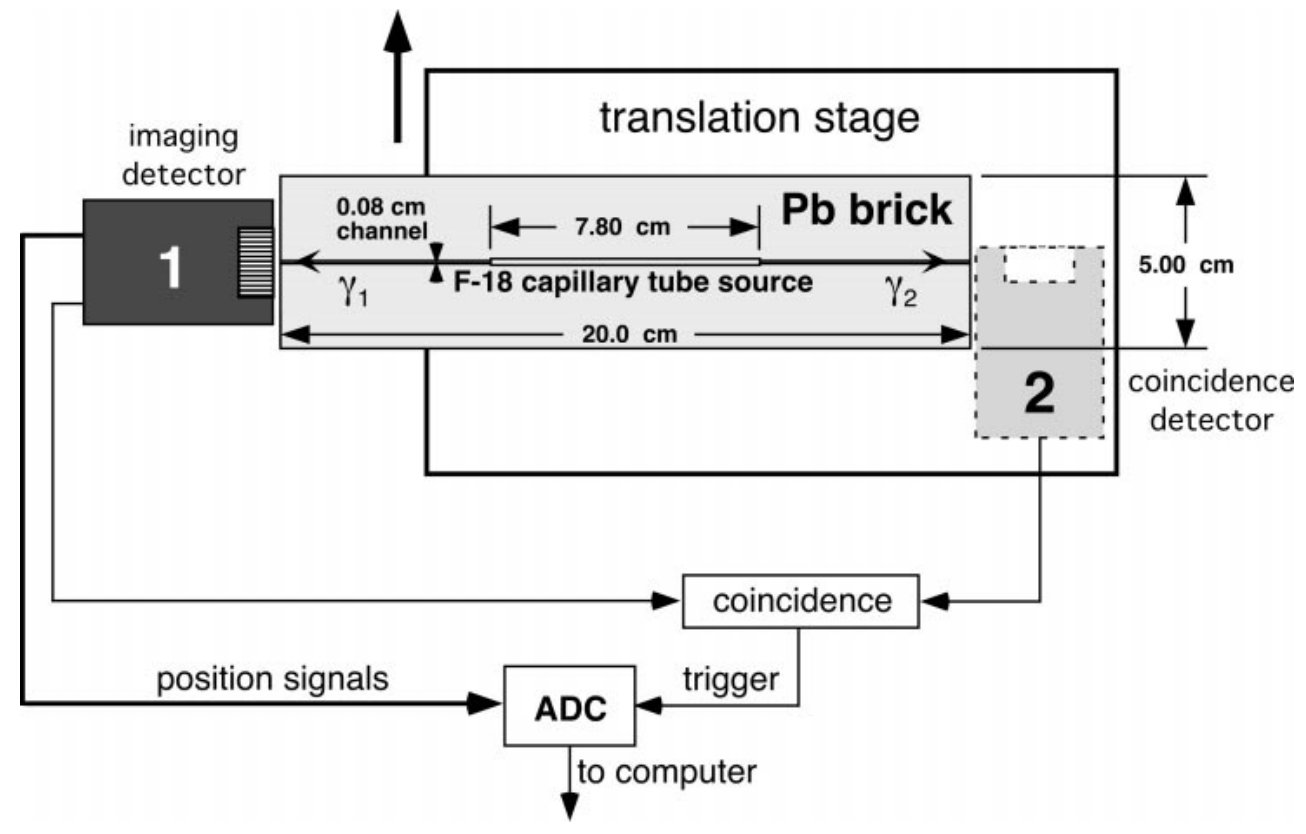

Fig. 2. Top view of scanning line source experiment.

sum fell within the specifie window, the event was accepted; otherwise it was rejected.

In addition to this "true" energy windowing, spatial exclusion criteria were also applied to the image data acquired in the scanning line source experiment described below. In these experiments, events were found to be positioned between apparent crystal centers in a regular pattern, as well as at the center of each crystal. In order to assess the effect of such intercrystal events on positioning accuracy, large (L) regions-ofinterest (ROI's) and small (S) ROI's were drawn around each crystal center. The large ROI's were created by the grid formed when the points midway between each apparent crystal center are connected to their nearest neighbors by (approximately) horizontal and vertical lines. The small ROI's were formed by placing irregularly shaped isocounts contours around each crystal center. Events occurring outside these small regions were rejected [15], [16]. The scanning line source data were then analyzed with the least restrictive exclusion conditions (L-spatial windowing and no energy windowing), with moderately restrictive exclusion conditions (L-spatial windowing and energy windowing), and the most restrictive conditions (S-spatial windowing and energy windowing).

\section{Event Positioning}

Different measures were employed to characterize the ability of the LSO/PSPMT detector module to identify the crystal-ofinteraction correctly when illuminated by single photon beams of widely differing energies.

First, the detector module was placed inside a $\mathrm{Pb}$ cave open on one side to accept radiation only from prepared sources. The module was then illuminated with single photons from a $1 \mathrm{~m}$ distant, small volume, high-activity radionuclide source emitting either $30-\mathrm{keV}$ (I-125), 140-keV (Tc-99m) or 511-keV (F-18) photons. The activity of these sources was chosen such that the module count rate due to the sources (approximately
30000 counts/s) was much higher than the count rate due to the LSO background ( 970 counts/s), yet low enough that the frequency of pileup events was negligible $(<1.2 \%$ of total). Images of the UFOV at these different energies and an LSO background image acquired with no external sources present were created using the centroid algorithm (1). Although the LSO background was low (about $3 \%$ of the total count rate), the LSO background image, scaled to the same imaging time, was subtracted from each floo image to remove the intrinsic LSO contribution.

Apparent crystal width in these floo images, expressed as a fraction of the true crystal width $(2 \mathrm{~mm})$, was estimated by measuring the FWHM and full-width-at-tenth-maximum (FWTM) of the count profile across each crystal in the central row of the LSO array. These values were averaged for each parameter. The largest and smallest apparent width values in this row were also determined.

Because detection of $511-\mathrm{keV}$ annihilation radiation may be an important application of this detector module, the detector response function at $511 \mathrm{keV}$ was also determined by scanning a highly collimated F-18 source across the detector module while in coincidence with a second LSO detector (Fig. 2). Although this measurement could, in principle, be carried out as a single photon experiment (since the beam was physically collimated), time coincidence was necessary to eliminate the LSO background. The collimator (described below) used to create the narrow photon beam reduced the photon flu from the F-18 source by a very large factor. Indeed, this reduction was so large that the detected singles rate in the module $(<20$ counts/s) was very small compared to the background count rate from LSO (970 counts/s) even when the highest available F-18 concentrations were used. Illuminating the module with a collinear, annihilation photon beam while in coincidence with a second detector eliminated the LSO background since Lu176 decays in the module are not correlated in time with those 

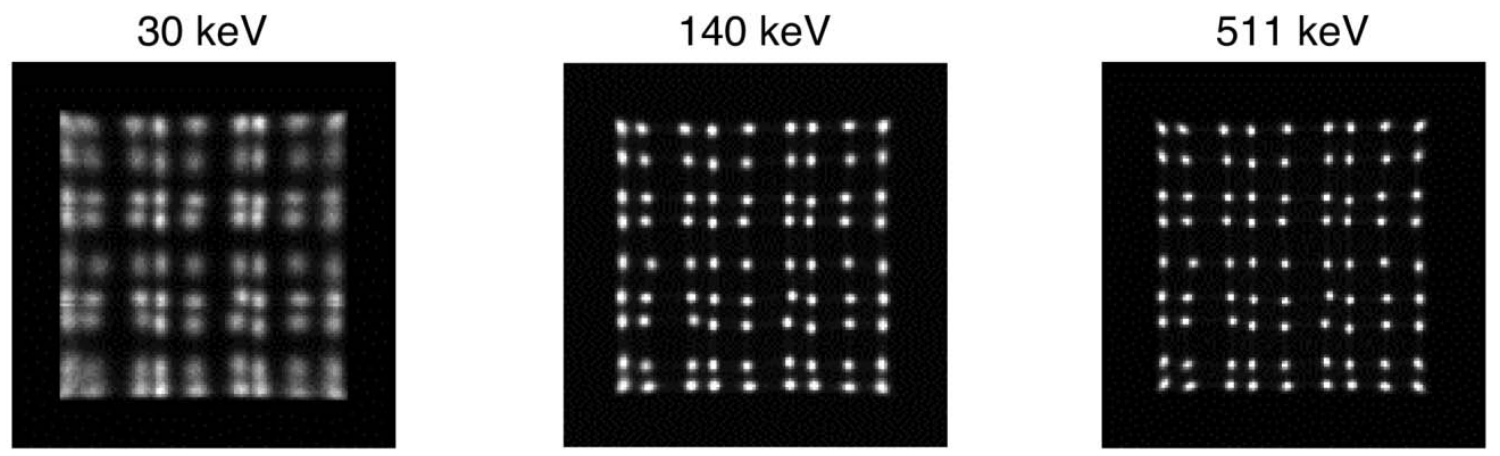

$\mathrm{I}-125$

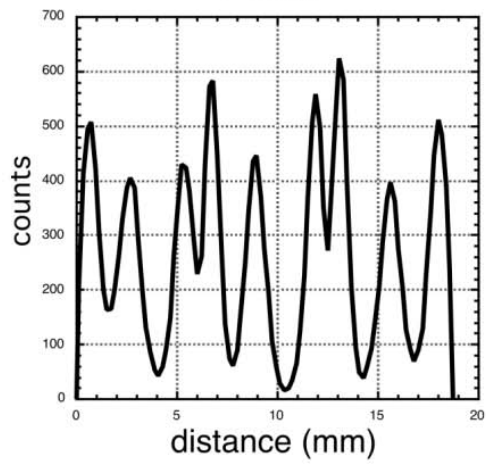

(a)

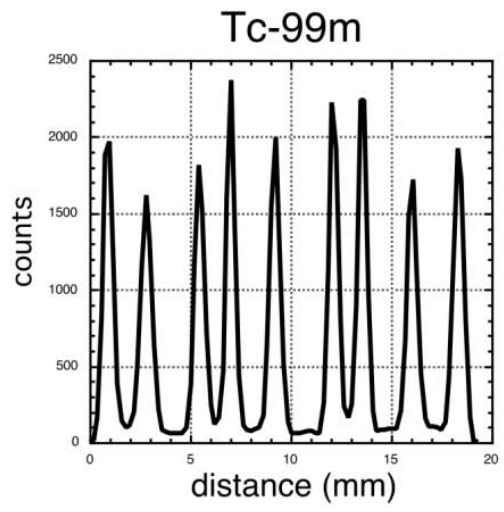

(b)

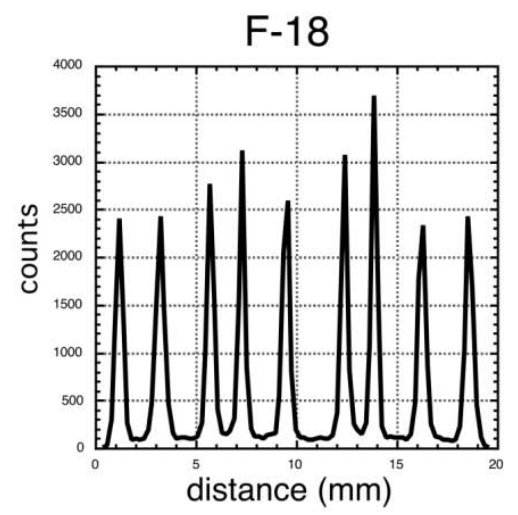

(c)

Fig. 3. (top) Images of the UFOV corrected for LSO background after fiel floo illumination with parallel beams of (single) photons of different energies. No event exclusion conditions were applied during creation of these images; (bottom) Profil across the central row of each corresponding figur in the upper panel. The distance across the UFOV between the centers of the extreme left and right columns is $17.4 \mathrm{~mm}$.

in the other detector. The signal-to-noise ratio of the measurement was markedly improved by this coincidence technique.

In order to produce a radiation beam narrow compared to the width of a crystal, a capillary tube (1.1-mm ID, 0.2-mm wall thickness, $75-\mathrm{mm}$ length) completely fille with F-18 was placed inside a $\mathrm{Pb}$ brick. The brick was machined in such a way as to allow two radiation beams, both narrower than the F18 flui column, to emerge from each end of the brick (Fig. 2). The capillary tube lay midway along a channel milled in each half of a brick so that when the two interlocking halves were placed together, the resulting channel formed a collimator hole running the length of the brick. In this configuration not less than $60 \mathrm{~mm}$ of $\mathrm{Pb}$ were interposed between the line source and either detector. The FWHM of these beams as they exited the brick was estimated to be approximately $0.8 \mathrm{~mm}$.

The brick, with source inside, was placed on a translation stage between the detector module and the second LSO detector such that one of the annihilation beams fell perpendicularly onto the face of the detector module while the second was incident on the other detector. By adjusting the position of the $\mathrm{Pb}$ brick on the stage, the beam directed onto the module was centered on the central row of crystals and stepped across the middle three crystals of this row in $0.25-\mathrm{mm}$ increments. At each position, an image of the FOV of the detector module was acquired. Detector response functions for the module were then created by plotting the fraction of accepted events assigned to each of the three crystals against source position.
Accuracy and sensitivity of event localization were computed from these same data. Accuracy (probability of correctly identifying the crystal-of-first-interaction or position detection accuracy [17]), was define as the counts occurring in a crystal when the radiation beam was located directly over the center of that crystal, divided by the counts assigned to crystals anywhere within the FOV. Sensitivity was define as the counts assigned to crystals divided by the number of counts assigned throughout the UFOV when the least restrictive spatial and energy selection criteria were applied. As noted in the Discussion, "accuracy" also measures the fraction of events whose position is not affected by intercrystal scattering.

\section{E. Spatial Linearity}

Spatial positioning linearity along the main diagonal of the background-corrected F-18 fiel floo image was evaluated by comparing apparent crystal separation along the diagonal to the known crystal separation. Measurements along the diagonal were chosen to reflec the (similar) $X$ and $Y$ spatial nonlinearities in a single data set.

\section{F. Gain Uniformity}

When a detector module is illuminated by a distant gamma ray-emitting source, the channel number of the photopeak maximum for each crystal measures apparent module gain at that location. Accordingly, a bar graph plot of the photopeak 


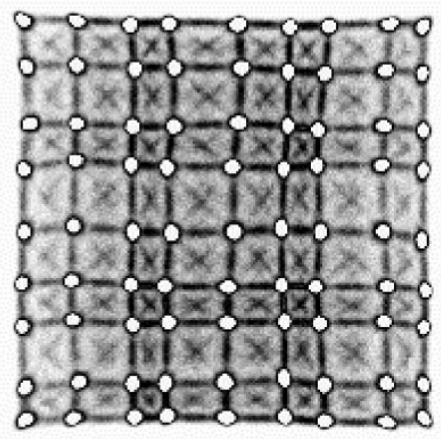

(a)

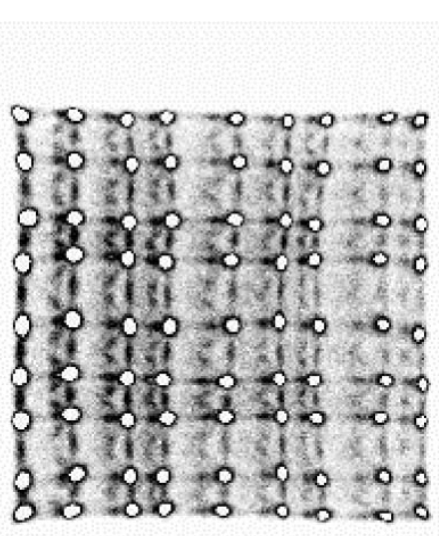

(b)

Fig. 4. Enhanced images highlighting the "connect-the-dot" pattern between crystals. Peak values are purposefully overexposed (white dots) to reveal faint structures (black) between crystals. (a) was obtained by illuminating the array from the front while (b) was obtained by illuminating the array from the left side.

maxima versus crystal location was constructed in order to visualize the spatial variation of module gain over the UFOV. Additional indirect measures of regional gain (apparent crystal width, energy resolution) were also tabulated.

\section{G. Energy Resolution}

Energy spectra were created for each crystal in the UFOV when illuminated with $511-\mathrm{keV}$ photons. Similarly, energy spectra were created using Tc-99m (140 keV) and I-125 ( 30 $\mathrm{keV}$ ) as the illumination sources. Since (single photon) spectra acquired in the presence of the LSO background may be distorted, spectra due to the LSO background were also created for each crystal in the UFOV with no external sources present and the detector module well shielded against external radiation. These LSO background spectra were subtracted before measurement of energy resolution. The gain normalization procedure was then applied to these data. The average energy resolution over the UFOV, the smallest and largest values of energy resolution within the UFOV, and the fraction of total LSO background counts occurring in the energy range $140 \pm$ $20 \mathrm{keV}$ were determined.

\section{H. Coincidence Timing}

Timing spectra were acquired using a TAC with and without time delay as described in Section II-B. Each of these timedisplaced distributions was fi with a Gaussian function and the FWHM determined from the fits These values were then converted to nanoseconds using the calibration factor determined from the time-displaced TAC's.

\section{RESULTS}

Single photon fiel floo images acquired at 30, 140, and $511 \mathrm{keV}$, and count profile along the central row of each image, are shown in Fig. 3. These images were created with the centroid algorithm (1) and are corrected for the LSO background. The least restrictive event selection criteria were applied during the creation of these images.

A version of Fig. 3(c), enhanced to reveal faint structures, is shown in Fig. 4(a). Each crystal in this image appears to be joined to its eight neighbors by faint horizontal, vertical, and diagonal straight lines in a "connect-the-dot" pattern. The details of this pattern change depending on whether the LSO array is illuminated from the front [Fig. 4(a)] or from the left side [Fig. 4(b)].

An LSO background image acquired with no external sources present, and a count profil along the central row, are shown in Fig. 5. The least restrictive event selection criteria were applied during creation of this image.

Average apparent crystal widths at the half maximum and tenth maximum levels, expressed as a fraction of the actual crystal width $(2 \mathrm{~mm})$, are listed in Table I for crystals in the central row of Fig. 3. Average widths, and the largest and smallest widths in the row, are included for images created from all data within the dynamic range of the ADC's. Average widths calculated from these same image data when subjected to energy windowing (W) are also shown. At $140 \mathrm{keV}$ the energy window was $140 \mathrm{keV} \pm 20 \mathrm{keV}$, and at $511 \mathrm{keV}$ the window was $511 \mathrm{keV} \pm 70 \mathrm{keV}$. Energy windowing was not applied to the I-125 fiel floo data since nearly all $30-\mathrm{keV}$ photon interactions in LSO are photoelectric and windowing is unnecessary.

The fraction of detected coincidence events that occur within each of three adjacent crystals scanned by the collimated F-18 line source is shown as a function of source position for different exclusion conditions in Fig. 6(a). The dotted curve portrays the fraction of events that occur in ROI's the size of an LSO crystal (L) when no energy exclusion condition is applied. The dashed curve portrays this fraction for events within the L-ROI and in the $511-\mathrm{keV} \pm 70-\mathrm{keV}$ energy range. The continuous curve portrays the fraction of events assigned to a crystal when events outside small (S) ROI's drawn around each apparent crystal are excluded, as well as events outside the energy window.

Accuracy and sensitivity are portrayed in the bar graph shown in Fig. 6(b) for different energy and spatial exclusion conditions when the scanning source is positioned directly over the center of the middle crystal in Fig. 6(a). For LROI's, energy exclusion conditions alone reduce the fraction of accepted events from $100 \%$ to $55 \%$, while the S spatial 


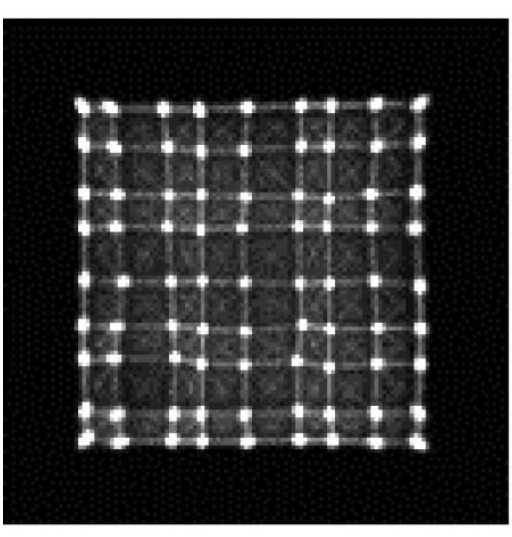

(a)
Central Row Profile

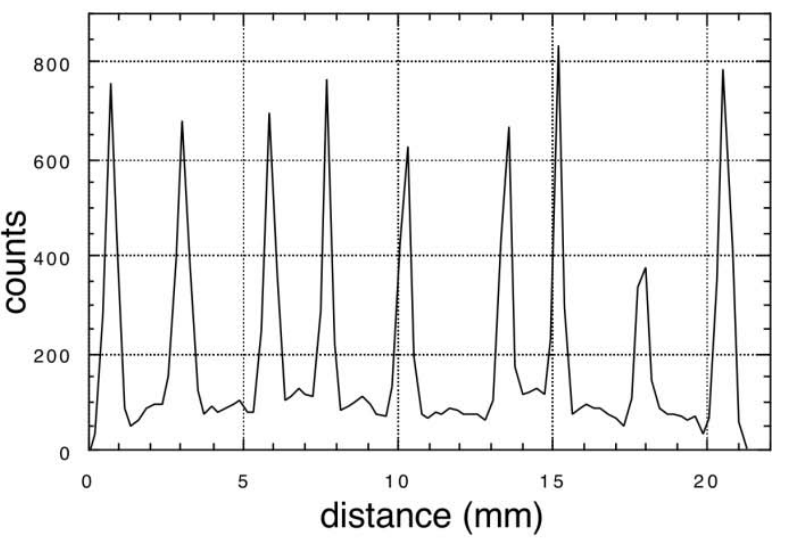

(b)

Fig. 5. (a) LSO background image; (b) Profil across central row of (a). The "connect-the-dot" pattern evident in Fig. 4 is also present in Fig. 4(a) although no external gamma ray sources are present.

TABLE I

Apparent Crystal Width ${ }^{1}$ Versus Photon Energy

\begin{tabular}{lccccc}
\hline \multicolumn{5}{c}{ Incident Photon Energy (keV) } \\
& 30 & 140 & $140(\mathrm{~W})^{3}$ & 511 & $511(\mathrm{~W})^{3}$ \\
\hline & & & & \\
FWHM $^{1}$ & 0.55 & 0.31 & 0.26 & 0.21 & 0.13 \\
& $(0.45-0.69)^{2}$ & $(0.27-0.35)$ & & $(0.15-0.27)$ & \\
FW IM $^{1}$ & -- & 0.60 & 0.50 & 0.45 & 0.29 \\
& & $(0.52-0.71)$ & & $(0.37-0.56)$ & \\
\hline
\end{tabular}

1: Expressed as a fraction of the true crystal width $(2 \mathrm{~mm})$; average of values from nine crystals in the central row of the LSO array.

2: Values in parentheses are the smallest - largest values in the central row

3 : $\mathrm{W}=$ energy windowed (see text).

exclusion condition combined with the energy exclusion condition reduces the fraction of accepted events further to $34 \%$. The accuracy of event positioning increases from $72 \%$ to $95 \%$ as these increasingly selective criteria are imposed.

The apparent separation between peaks along the main diagonal of Fig. 3(c) is plotted in Fig. 7(a) where the actual diagonal crystal separation is $3.07 \mathrm{~mm}$. The largest deviation of peak separation from the mean peak separation was $0.82 \mathrm{~mm}$, while the average deviation from the mean peak separation was $0.6 \mathrm{~mm}$. The sinusoidal-like variation in spacing between peaks in Fig. 7(a) is detectable in Figs. 3, 4, and 5(a).

The TAC spectrum obtained with the detector module in time coincidence with a second LSO detector is shown in Fig. 9. The second timing peak was acquired with an interposed time delay of 4 ns. The average FWHM of these peaks was $1.2 \mathrm{~ns}$.

\section{DISCUSSION}

\section{A. Event Positioning}

Fig. 3 illustrates that individual LSO crystals are well resolved at 140 and $511 \mathrm{keV}$, and identifiable separately, even at $30 \mathrm{keV}$. The apparent widths of each crystal (Table I) and the high peak-to-valley ratios evident in the profile shown in Fig. 3 (greater than 20-to-1 at $511 \mathrm{keV}$ ), suggest that individual crystals can be identifie with high contrast down to at least $140 \mathrm{keV}$ and perhaps lower. That individual crystals can still be identifie at $30 \mathrm{keV}$ implies that the combination of LSO, surface treatment, direct crystal-PSPMT coupling and the performance of the PSPMT itself, is an efficien mechanism for locating absorption events within the scintillator array. Indeed, all of the apparent crystal widths listed in Table I for Tc-99m and F-18, both FWHM and FWTM, are much smaller than the width of the real LSO crystals $(2 \mathrm{~mm})$. These results suggest that the cross-sectional width of the LSO crystals could be further reduced, thereby allowing fine spatial sampling while still maintaining good event positioning [17], [18].

The ability to identify crystals as separate from one another is a necessary, but not sufficien condition, to claim correct identificatio of the crystal-of-first-interaction With L-ROI spatial windowing, the probability of correctly identifying the crystal-of-first-interactio at $511 \mathrm{keV}$ is, according to Fig. 6(b), approximately $72 \%$, and rises only slightly, if at all, as the energy threshold is increased to $440 \mathrm{keV}$. This result is in general agreement with the simulation results obtained by Shao et al. [17] for an LSO array with pitch, crystal size, and reflecto nearly identical to that studied here, although Shao et al. observed a slight decline in accuracy with increasing energy threshold. The relative independence of accuracy from energy threshold also agrees closely with LSO simulation results obtained by Moisan et al. [19] despite differences in simulated crystal size, surface treatment, and readout method.

The accuracy estimates shown in Fig. 6(b) for the S-ROI exclusion condition, however, are higher than reported by either of these investigators ( $>90 \%$ here versus $82 \%$, average maximum value) for either BGO or LSO. Since the S-ROI excludes events positioned between crystal centers, this improvement must be due to elimination of the connect-the-dot pattern shown in Fig. 4. It is of interest, therefore, to identify the mechanism by which events are positioned in this pattern. 


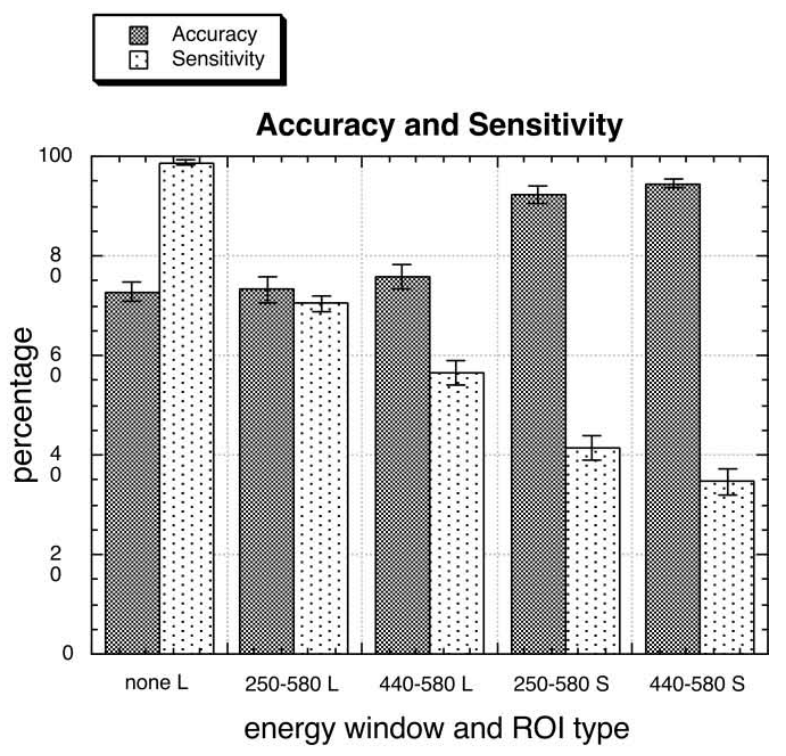

(b)

Fig. 6. (a) Fraction of all accepted events occurring in each of three adjacent, central row LSO crystals as a highly collimated beam of normally incident annihilation photons is scanned across the detector module while in time coincidence with a second LSO detector; dotted curve: no event exclusion conditions; dashed curve: energy and L-ROI windowing; continuous curve: energy and S-ROI windowing. (b) Accuracy and sensitivity calculated for source positions in which the beam falls entirely within the center crystal in (a) (see text).

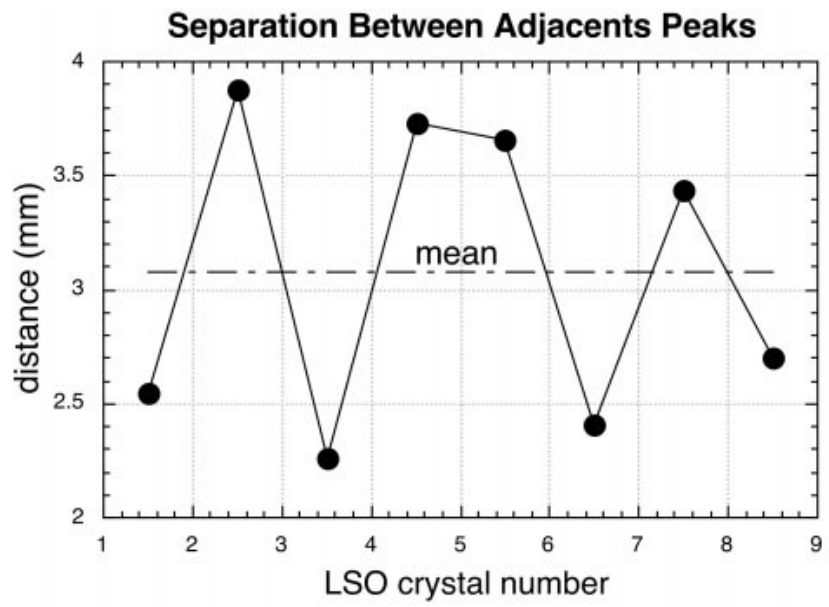

(a)

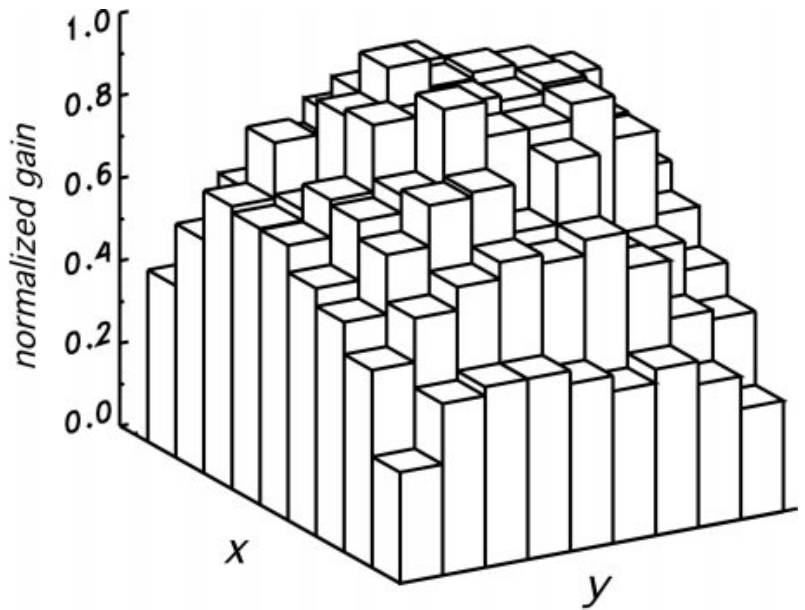

(b)

Fig. 7. (a) Apparent crystal separation versus position along the main diagonal of Fig. 3(c). Peak separation is not constant and appears to depend on the position of the LSO crystals relative to the position of the PSPMT anode plates. (b) Normalized module gain versus crystal position within the UFOV.

Fig. 7(b) shows apparent module gain before correction as a function of crystal location. Gain is define as the normalized channel number in which the photopeak maximum occurs for each crystal.

Energy spectra for three different incident photon energies are shown in Fig. 8 for the same central crystal. These spectra have been corrected for the LSO background, whose spectrum is shown in Fig. 8(c) The fraction of LSO background events occurring in the energy range $140 \pm 20 \mathrm{keV}$ [Fig. 8(c)] was $1.2 \%$ of the total LSO background rate or about 12 counts/s.

Energy resolution and its variation within the UFOV are listed in Table II for several different incident photon energies. For illustrative purposes, energy resolution at $511 \mathrm{keV}$ in an identical detector module composed of $2 \mathrm{~mm} \times 2 \mathrm{~mm} \times 10$ mm-long BGO crystals rather than LSO crystals is also included.

If an incident photon scatters from one LSO crystal into a nearby crystal and is absorbed in that crystal, the centroid algorithm will position this event along a line joining the two crystal centers. The frequency with which events are positioned in a given line will depend on the probability of the scattered photon reaching the second crystal and being absorbed in that crystal, while the position of the event along the line will be determined by the relative amounts of energy deposited in the two crystals. If many of these kinds of events are recorded, a spatial pattern, or image, should emerge 


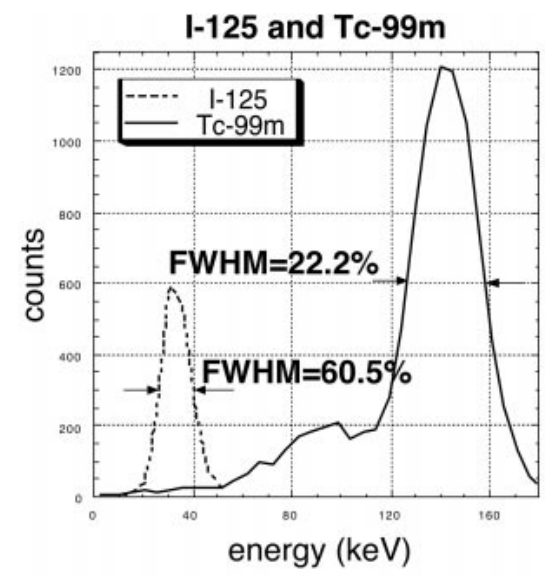

(a)

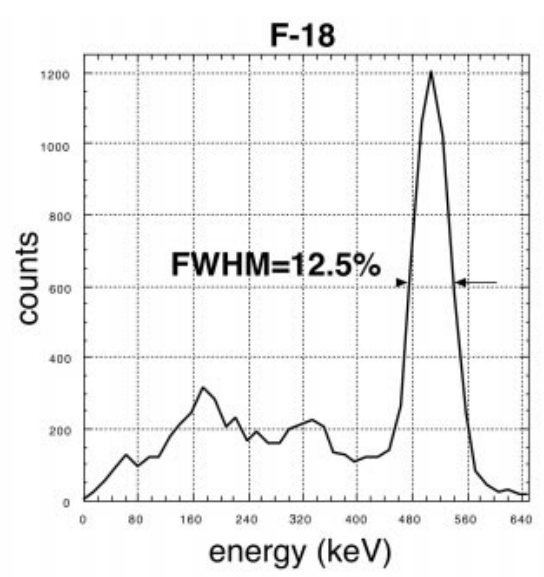

(b)

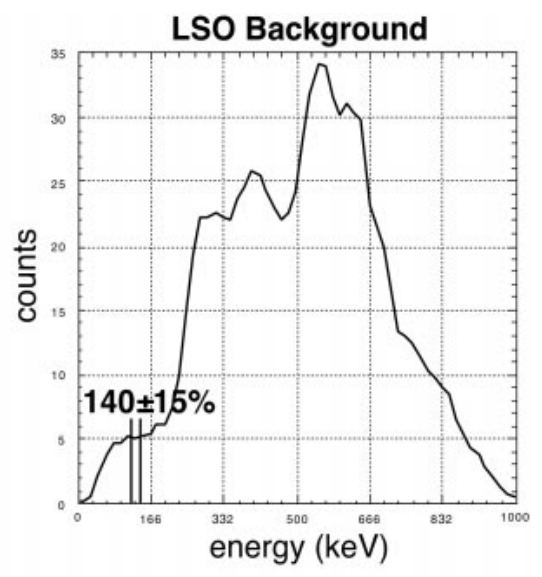

(c)

Fig. 8. Energy spectra for the same central crystal when exposed to parallel beams of single photon radiation of (a) 30 and $140 \mathrm{keV}$, (b) $511 \mathrm{keV}$, and (c) with no external sources of radiation present (the Lu-176 background spectrum). Note the location of the emission region of Tc-99m superimposed on this spectrum.

TABLE II

Energy Resolution ${ }^{1}$ Versus Photon Energy

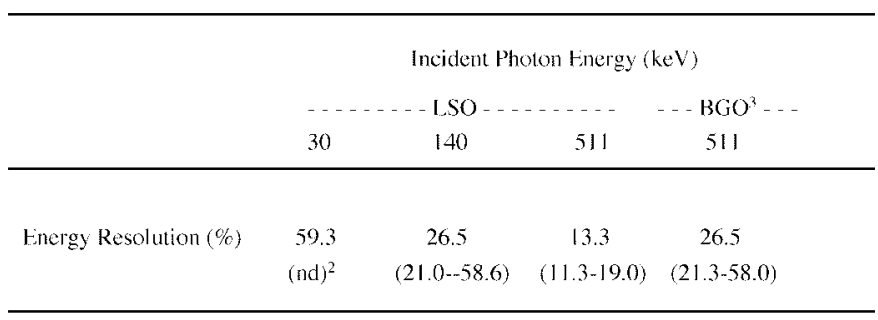

1: Average energy resolution over the full UFOV after correction for gain variations.

2: Values in parentheses are the smallest-largest values observed anywhere within the

UPOV after correction for gain variations; nd= not determined.

3: Energy resolution in an identical detector modulc but composed of $2 \mathrm{~mm} \times 2 \mathrm{~mm} \times$ $10 \mathrm{~mm}$ long BGO crystais

in which events are arranged along horizontal, vertical, and diagonal straight lines that appear to connect crystal centers. This is, in fact, the general appearance of the pattern shown in Fig. 4(a).

If this explanation is correct, then the frequency of occurrence of events in a particular line should depend on several factors. For example, more events should be positioned in the vertical and horizontal lines in Fig. 4(a) than along the diagonals. This result is expected since crystals with sides in contact with a given crystal present a larger solid angle to photons scattered from that crystal than crystals touching only at the corners. Further, only the main diagonals through each crystal should be easily detected, while second-order diagonals should not. This result is expected since a substantial amount of LSO must be traversed by a scattered photon in order to interact in a distant, nonmain diagonal crystal. Because the energy of the scattered photon is lower than $511 \mathrm{keV}$, and the absorption probability is correspondingly increased, nonmain diagonal interactions should occur with low probability. Thus, the second-order pattern should be much fainter than the pattern generated by nearest-neighbor interactions. Both of these predictions are borne out in Fig. 4(a).

\section{TAC Spectrum (LSO)}

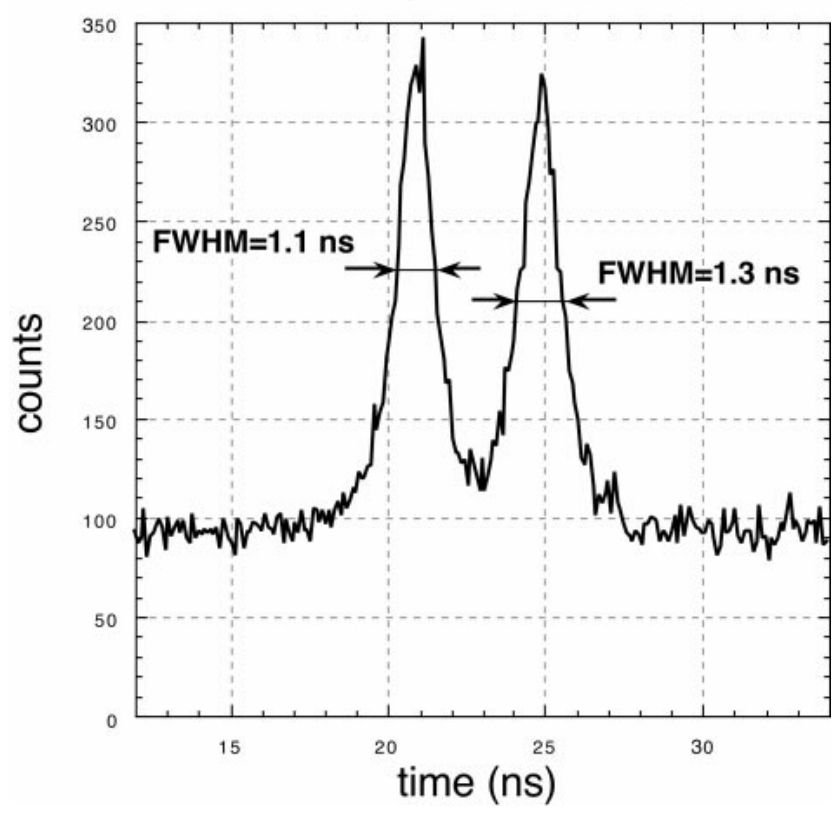

Fig. 9. TAC spectrum obtained with the detector module in time coincidence with a second LSO detector. The second peak in the spectrum was created by introducing a four nanosecond time delay. The singles rate on each detector was 300000 counts per second in this experiment.

The pattern should also change when the module is illuminated from the side rather than the front. Front-on illumination should cause the connect-the-dot pattern to be symmetric around each crystal center [Fig. 4(a)], except for the edge crystals, because scattering angles around the normal to the array are equiprobable. In contrast, side illumination should cause the pattern to exhibit an asymmetry along the direction of illumination but remain symmetric in the direction perpendicular to the direction of illumination. With side illumination, the pattern should depend on the product of the scattering angle probability distribution and the energy-dependent absorption probability of the scattered photon along the photon fligh path. Since forward scattered radiation is more energetic 
than backscattered radiation, and LSO has a rapidly varying attenuation coefficien with photon energy, the combination of these distributions should be asymmetric along the direction of the incident radiation. An asymmetry of this kind is, in fact, evident in Fig. 4(b) when the module is illuminated from the left side. The pattern on the left side of a crystal center differs from that on the right side of the crystal center, whereas the patterns above and below a crystal center are the same. These results strongly suggest, therefore, that the connectthe-dot pattern is a direct spatial manifestation of intercrystal scatter that provides a means of distinguishing photons totally absorbed in the crystal of firs interaction from photons that are firs scattered and then totally absorbed in another crystal in the LSO array.

The efficac of the S-ROI and photopeak energy selection conditions in eliminating all but total absorption events in the crystal of firs interaction can be further tested by asking what is the highest possible sensitivity (not accuracy) that can be achieved under the experimental conditions. If the energy and S-ROI acceptance conditions do, in fact, retain only firs crystal total absorption events, then the highest possible sensitivity should be comparable to the photofraction of LSO. In the classic narrow beam, thin sample, geometry necessary for such measurements, the photofraction of a material is the fraction of incident photons that undergo complete absorption on their firs interaction. The photofraction of LSO at 511 $\mathrm{keV}$ is 0.33 [11], [12], [20] a value very near the sensitivity estimate shown in the far right bar of Fig. 6(b) (0.34). This agreement provides independent verificatio that nearly all photons scattered out of the crystal of firs interaction are eliminated by the two exclusion conditions.

The existence of this scatter-dependent, connect-the-dot pattern is of interest for several reasons. A variety of studies, including the present one, have shown that the position detection accuracy of LSO is similar to BGO when only energy windowing is used to accept or reject events. This result has also been interpreted to mean that the higher light output of LSO does not confer any additional improvement in accuracy compared to BGO [17], [19]. The present study suggests that, at least in principle, this is not the case. The much higher light output of LSO, combined with efficien collection and photoelectron conversion of this light by the PSPMT, permits total absorption events in the crystal-offirst-interactio to be spatially discriminated from intercrystal scatter-total absorption events even though both types of events are energetically identical. Since scatter-total absorption events comprise a significan fraction of all interactions in both LSO and BGO detectors [19], and these events are frequently mispositioned [15], the ability to eliminate or reduce the number of these events in LSO should be a potential advantage of $\mathrm{LSO}$ relative to $\mathrm{BGO}$.

The connect-the-dot pattern should be present in all geometrically similar scintillator arrays. However, the ability to detect the pattern likely depends on the light output of the scintillator and the efficienc of the light collection/photoelectron conversion process. Although the degrading effects of scatter within crystal arrays have been studied and are understood, we are unaware of any reports of this pattern in modules comprised of $\mathrm{BGO}$ [15], suggesting that the underlying pattern is obscured by the poor photostatistics generally associated with BGO modules. Similarly, we are unaware of experimental or simulation studies of LSO detector modules that report the connect-the-dot pattern, although this pattern should emerge naturally if the simulated light collection/photoelectron conversion efficienc is high enough and if all relevant physical processes are modeled.

The present study possesses several limitations with regard to the measurement of accuracy and in the assessment of the relationship between accuracy and other variables. For example, accuracy is likely somewhat overestimated since the beam diameter in the scanning line source experiment is smaller than the crystal width. A "true" estimate of accuracy would require that the incident beam be exactly the size of the crystal cross section and aligned with this cross section.

Further study is also required to defin more fully the dependence of accuracy and sensitivity on the size and shape of the S-ROI, and the degree to which changes in this ROI affect coincidence resolution, a variable known to be weakly dependent on intercrystal scatter [17].

Finally, it may be that some portion of the scattered events that appear in the connect-the-dot pattern, and that are rejected by the spatial window, can be "recovered" [21], [22] by exploiting details of the scatter interaction-spatial positioning process not considered in this work.

\section{B. Spatial Linearity}

The variation in apparent crystal separation along a diagonal [Fig. 7(a)] is a significan fraction of the mean crystal separation. The module possesses, therefore, a distinct spatial nonlinearity in event positioning. The undulating separation of rows and of columns evident in Figs. 3-5 suggests that some underlying mechanism is present that causes the same spacing anomaly to occur in both coordinate directions. Similar nonlinearities have been reported previously [23].

The most likely explanation for this effect is spatial undersampling of the electron cloud by the $4 X$ anode and $4 Y$ anode plate structure of the PSPMT. Assume, for example, that a small diameter light pulse is projected onto the photocathode of the PSPMT and that the amplifie electron cloud, assumed to have a Gaussian charge distribution in cross-section, exhibits an effective diameter about twice the anode spacing. If the light pulse is incident midway between an anode pair, large and roughly equal amounts of charge will be accumulated on each anode. In this case the calculated event positions will be a sensitive function of the true position of the source and small movements of the pulse source will cause large displacements in apparent position. In contrast, if the pulse source is moved directly over an anode plate, the two adjacent plates will accumulate only small amounts of charge. Most of the charge will be collected on the plate under the event. In the firs case, positioning will be sensitive to small source displacements and apparent displacement will follow closely true displacement. In the second case, however, the same movement of the light source will produce smaller displacements in apparent position. This model predicts, therefore, that if the anode array 
actually does effectively undersample the electron cloud, the spacing between columns and between rows will be decreased for events occurring over anode plates and increased for events occurring between anode plates.

Figs. 3-5 show that apparent row and column separations agree with this prediction. Moving away from the image center, apparent crystal separation shrinks as the center of the firs anode is approached, then expands in the next interplate gap, then shrinks again as the outer anode plate is approached. This result implies that 4 anode plates in each coordinate direction are too few to produce uniform spatial linearity.

We should note that spatial linearity could be improved by increasing the size of each crystal in the array while still covering the UFOV, or by increasing the thickness of the PSPMT glass entrance window. Two mm square LSO crystals coupled to a thin entrance window cast a relatively small light spot onto the PSPMT photocathode, thereby approximating the small projected light spot described above. However, if the cross-sectional area of each crystal were increased and/or window thickness increased, scintillation light would be spread over a larger area of the photocathode and the associated electron distribution would be spread more uniformly over adjacent anode plates. While this scheme would improve spatial linearity, it would also degrade the spatial localization properties of the module, an unacceptable trade-off if highresolution imaging is the goal. In the present case, this spatial nonlinearity is more of an inconvenience than a limitation. Since each LSO crystal is readily distinguished from its neighbors, the location of a crystal-of-interaction in "real" space can be readily obtained by lookup table since the true location of each crystal in the LSO array is known with high accuracy.

\section{Gain Uniformity}

Fig. 7(b) illustrates that apparent gain decreases from the center to the edges of the UFOV and is lowest in the corners. The absolute gain, however, appears large enough that event positioning (Fig. 3) and apparent crystal width (Table I) are not unduly compromised even at these locations. The regional gain variations evident in Fig. 7(b) require correction if global lower and upper energy thresholds are to be used over the entire UFOV (as was done here). This scaling, while compensating for any crystal-to-crystal variations in LSO light output [24], regional differences in optical coupling efficienc or regional PSPMT amplificatio variations, does not correct for variations in energy resolution from crystal-to-crystal and a different correction would be needed for this purpose.

\section{Energy Resolution}

Energy resolution at 30-, 140-, and 511-keV [Table II, Fig. 8(a)-(b)] implies that the LSO/PSPMT combination is an efficien means of creating, transferring, collecting, and amplifying scintillation light. Moreover, LSO appears to possesses a scintillation efficienc about four times greater than BGO based on the square of the ratio of LSO to BGO energy resolution at $511 \mathrm{keV}$ (Table II). This value is somewhat higher than the ratio of absolute light outputs of these two scintillators (3.2) calculated from the data of Moszynski et al. [24]. However, the present experiment tends to underestimate BGO light output relative to LSO by ignoring differences between these scintillators that affect light collection efficiency e.g., differences in emission spectra, etc. Correction for these differences would tend to reduce the observed ratio to a value nearer the ratio of absolute light outputs.

Apparent crystal width at $511 \mathrm{keV}(\mathrm{W})(\mathrm{FWHM}=0.13$, Table I) predicts the apparent crystal width at $30 \mathrm{keV}(0.54$ calculated, 0.55 measured) and at $140 \mathrm{keV}(\mathrm{W})(0.25$ calculated, 0.26 measured) when scaled by the square root of the ratio of incident gamma ray energies [25], [26]. These results suggest the internal consistency of these data and imply that factors associated with the creation and collection of scintillation light dominate the detection process.

These results also support the view that the exact location of the point of gamma ray absorption within a crystal does not strongly influenc the fraction of scintillation light reaching the photocathode of the PSPMT. Because of the very high linear attenuation coefficien of LSO at low photon energies, nearly all $30-\mathrm{keV}$ photons will be absorbed within the firs millimeter, and nearly all $140-\mathrm{keV}$ photons within the firs $3 \mathrm{~mm}$, of the 10 -mm-long crystal. In contrast, somewhat less than $60 \%$ of $511-\mathrm{keV}$ photons will interact anywhere along the entire 10$\mathrm{mm}$ crystal length. If the fraction of scintillation light reaching the photocathode of the PSPMT were a strong function of the point of the interaction within the crystal, the apparent size of the crystal at 30 and $140 \mathrm{keV}$ would not be predicted by the apparent crystal size at $511 \mathrm{keV}$. Thus, light collection appears to be relatively independent of the gamma ray interaction site or, equivalently, of incident gamma ray energy.

\section{E. LSO Background}

The long-lived $\left(3.1 \times 10^{10}\right.$ years $)$ background radioactivity of LSO creates an image of the FOV when no external radioactivity is present (Fig. 5). Lu-176 decays by beta emission (100\%) accompanied by sequential emission of a $306-\mathrm{keV}$ gamma ray $(95 \%)$, a $202-\mathrm{keV}$ gamma ray $(85 \%)$, and an 88 $\mathrm{keV}$ gamma ray $(15 \%)$ for a total decay energy (Q value) of $1026 \mathrm{keV}$ [27]. This combination of Lu emissions creates, in turn, an intrinsic energy spectrum [Fig. 8(c)] consisting of a superposition of the continuous electron energy distribution with the gamma rays, their escape peaks and other internal emissions. The distribution of detected events is broad (as expected), with a maximum between 500 and $600 \mathrm{keV}$. Although the effects of this background can be removed by placing paired LSO detector modules in time coincidence, single photon imaging with these modules will be affected. Fig. 8(c) indicates that the degree of background contamination in a single photon study will be a function of photon energy and the width of the selected energy window. For single photon energies above about $200-\mathrm{keV}$, background contamination will be relatively large while for energies below about $200-\mathrm{keV}$ contamination will be relatively small. The background rate in the $140 \pm 15 \%$ region is low enough that background subtraction effectively eliminates the Lu-176 contribution. It should be noted, however, that this low rate is due not only to 
the low LSO background rate in the Tc-99m region of the LSO energy spectrum, but also to the low total mass of LSO in the detector module. If detectors with larger masses of LSO were operating at higher single photon energies, two deleterious effects would occur. First, the background count rate within the selected single photon energy window would increase and, second, the total event rate to which the detector module is exposed would also increase, potentially adding a significan dead-time burden to the acquisition electronics. In the present case, neither the total event rate nor the rate occurring in the Tc region of the LSO spectrum present significan difficulties but large area (volume) detectors would require methods to suppress or, otherwise, correct for these effects. LSO arrays intended specificall for low-energy use could also be made much thinner than the module evaluated here, yet still stop a large fraction of incident photons. In this case, the LSO background rate could be reduced further.

The connect-the-dot pattern created when the detector module is illuminated with $511 \mathrm{keV}$ is also present in the LSO background image [Fig. 5(a)] even when no external radiation is present. In this case, however, the pattern is created by a combination of electron absorption in the crystal of Lu-176 disintegration followed by absorption of an escape gamma ray in a nearby crystal. It is not difficul to show that this process will also gives rise to a symmetric connect-the-dot pattern similar to that observed with front-on external illumination [Fig. 4(a)].

\section{F. Coincidence Timing}

The TAC spectrum portrayed in Fig. 9 indicates that coincidence timing of the LSO/PSPMT detector module (FWHM = $1.2 \mathrm{~ns}$ ) is favorable when compared with detector modules using BGO. The intrinsic timing response of the PSPMT (anode pulse rise time $=1.4 \mathrm{~ns}$, [13]) and the large and very rapidly rising light pulse of LSO yield FWHM values that are a factor of two or more smaller than reported for BGO [10]. LSO/PSPMT modules in time coincidence should, therefore, provide more effective discrimination against random coincidences.

\section{CONCLUSIONS}

Compact detector modules, created by coupling arrays of small, individual crystals of LSO directly to miniature metalcan PSPMT's, offer a means of detecting and accurately locating the crystal-of-interaction over a range of photon energies. At $511 \mathrm{keV}$, event positioning accuracy does not improve significantl with energy windowing, but does improve with spatial windowing by eliminating totally absorbed intercrystal scatter events that are positioned between crystal centers. The module, while possessing good energy resolution and an increased ability to reject random coincidences, also exhibits a distinct spatial positioning nonlinearity and a spatial variation in gain.

The Lu-176 background, less important in coincidence detection, can interfere with single photon imaging. However, the background rate in the photon energy range associated with many conventional single photon imaging studies, i.e., with Tc-99m, is small and should be correctable for low-mass LSO arrays.

Together, these results suggest that the LSO/PSPMT detector module may be of use in applications requiring highperformance in a compact device, applications that range from hand-held imaging probes to full ring PET scanners for small animals.

\section{ACKNOWLEDGMENT}

The authors wish to thank J. V. Sullivan, R. W. Seldon, and B. E. Chidakel for their expert assistance in preparing the mechanical and electrical components of the detector modules.

\section{REFERENCES}

[1] R. R. Raylman, S. J. Fisher, R. S. Brown, S. P. Either, and R. L. Wahl, "Fluorine-18-fluorodeoxyglucose-guide breast cancer surgery with a positron-sensitive probe: Validation in preclinical studies," J. Nucl. Med., vol. 36, pp. 1869-1874, 1995.

[2] N. E. Hartsough, H. H. Barrett, H. B. Barber, and J. M. Woolfenden, "Intraoperative tumor detection: Relative performance of single-element, dual-element and imaging probes with various collimators," IEEE Trans. Med. Imag., vol. 14, pp. 259-265, 1995.

[3] M. P. Tornai, L. R. MacDonald, C. S. Levin, S. Siegel, and E. J. Hoffman, "Design considerations and initial performance of a $1.2 \mathrm{~cm}^{2}$ beta imaging intra-operative probe," IEEE Trans. Nucl. Sci., vol. 43, pp. 2326-2335, 1996.

[4] B. E. Patt, M. P. Tornai, J. S. Iwanczyk, C. S. Levin, and E. J. Hoffman, "Development of an intraoperative gamma camera based on a 256-pixel mercuric iodine detector array," IEEE Trans. Nucl. Sci., vol. 44, pp. 1242-1248, 1997.

[5] M. Watanabe, H. Uchida, H. Okada, K. Shimizu, N. Satoh, E. Yoshikawa, T. Ohmura, T. Yamashita, and E. Tanaka, "A high-resolution PET for animal studies," IEEE Trans. Med. Imag., vol. 11, pp. 577-580, 1992.

[6] P. D. Cutler, S. R. Cherry, E. J. Hoffman, W. M. Digby, and M. E. Phelps, "Design features and performance of a PET system for animal research," J. Nucl. Med., vol. 33, pp. 595-604, 1992.

[7] R. Lecomte, J. Cadorette, P. Richard, S. Rodrigue, and D. Rouleau, "Design and engineering aspects of a high-resolution positron tomograph for small animal imaging," IEEE Trans. Nucl. Sci., vol. 41, pp. 1446-1452, 1994.

[8] S. R. Cherry, Y. Shao, R. W. Silverman, K. Meadors, S. Siegel, A. Chatziioannou, J. W. Young, W. F. Jones, J. C. Moyers, D. Newport, A. Boutefnouchet, T. H. Farquhar, M. Andreaco, M. J. Paulus, D. M. Binkley, R. Nutt, and M. E. Phelps, "MicroPET-A high-resolution PET scanner for imaging small animals," IEEE Trans. Nucl. Sci., vol. 44, pp. 1161-1166, 1997.

[9] O. Fries, S. M. Bradbury, J. Gebauer, I. Holl, E. Lorenz, D. Renker, and S. I. Ziegler, "A small animal PET prototype based on LSO crystals read-out by avalanche photodiodes," Nucl. Instrum., Meth. in Phys. Res., vol. 387, pp. 220-224, 1997.

[10] M. Watanabe, T. Omura, H. Kyushima, Y. Hasegawa, and T. Yamashita, "A compact position-sensitive detector for PET," IEEE Trans. Nucl. Sci., vol. 42, pp. 1090-1094, 1995.

[11] C. L. Melcher and J. S. Schweitzer, "Cerium-doped lutetium oxyorthoslicate: A fast, efficien new scintillator," IEEE Trans. Nucl. Sci., vol. 39, pp. 502-505, 1992.

[12] J. Seidel, W. R. Gandler, and M. V. Green, "Characteristics of a pair of small-field-of-vie LSO scintillation cameras," IEEE Trans. Nucl. Sci., vol. 43, pp. 1968-1973, 1996.

[13] K. K. Hamamatsu-Photonics, "Position sensitive photomultiplier tube R5900-00-C8 and R5900U-00-C8," in Data Sheet, Japan, 1996.

[14] M. E. Casey, L. Eriksson, M. Schmand, M. S. Andreaco, M. Paulus, M. Dahlbom, and R. Nutt, "Investigation of LSO crystals for high-spatialresolution positron emission tomography," IEEE Trans. Nucl. Sci., vol. 44, pp. 1109-1113, 1997.

[15] P. D. Cutler and E. J. Hoffman, "Use of digital front-end electronics for optimization of a modular PET detector,' IEEE Trans. Med. Imag., vol. 13, pp. 408-418, 1994.

[16] J. Seidel, S. Siegel, W. R. Gandler, and M. V. Green, "Accuracy of event positioning in a pixelated BGO array coupled to a miniature position sensitive phototube," J. Nucl. Med., vol. 38, p. 197P, 1997. 
[17] Y. Shao, S. R. Cherry, S. Siegel, and R. W. Silverman, "A study of intercrystal scatter in small scintillator arrays designed for high-resolution PET imaging," IEEE Trans. Nucl. Sci., vol. 43, pp. 1938-1944, 1996.

[18] C. E. Ordonez, W. Chang, J. Liu, and D. L. Gunter, "Simulation of light output from narrow sodium iodine detectors," IEEE Trans. Nucl. Sci., vol. 44, pp. 1237-1241, 1997.

[19] C. Moisan, D. Vozza, and M. Loope, "Simulating the performances of an LSO based position encoding detector for PET," IEEE Trans. Nucl. Sci., vol. 44, pp. 2450-2458, 1997.

[20] F. Daghighian, P. Shenderov, K. S. Pentlow, M. C. Graham, B. Eshaghian, C. L. Melcher, and J. S. Schweitzer, "Evaluation of cerium doped lutetium oxyorthosilicate (LSO) scintillation crystal for PET," IEEE Trans. Nucl. Sci., vol. 40, pp. 1045-1047, 1993.

[21] R. Lecomte, C. Martel, and J. Cadorette, "Study of the resolution performance of an array of discrete detectors with independent readouts for positron emission tomography," IEEE Trans. Med. Imag., vol. 10, pp. 347-357, 1991.
[22] K. A. Comanor, P. R. G. Virador, and W. W. Moses, "Algorithms to identify detector compton scatter in PET modules," IEEE Trans. Nucl. Sci., vol. 43, pp. 2213-2218, 1996.

[23] R. Pani, A. Pergola, R. Pellegrini, and A. Soluri, "New generation position-sensitive PMT for nuclear medicine imaging," Nucl. Instrum., Meth. in Phys. Res., Section A (Accelerators, Spectrometers, Detectors, and Associated Equipment), vol. 392, pp. 319-323, 1997.

[24] M. Moszynski, M. Kapusta, M. Mayhugh, D. Wolski, and S. O. Flyckt, "Absolute light output of scintillators," IEEE Trans. Nucl. Sci., vol. 44, pp. 1052-1061, 1997.

[25] R. Kurz, R. Reinartz, S. Widdau, J. Schelten, A. Scholz, and W. Schäfer, "Two-dimensional neutron detector based on a position-sensitive photomultiplier," Nucl. Instrum., Meth. in Phys. Res., vol. A273, pp. 273-282, 1988.

[26] M. Green and J. Seidel, "Single photon imaging," in Nuclear Medicine Diagnosis and Therapy, J. Harbert, W. Eckelman, and R. Neumann, Eds. New York: Thieme Medical, 1996, pp. 87-120.

[27] C. M. Lederer, J. M. Hollander, and I. Perlman, Table of Isotopes, 6th ed. New York: Wiley, 1967. 